\title{
Fisioterapia no pós-operatório de Tetralogia de Fallot
}

A Tetralogia de Fallot é uma cardiopatia congênita cianótica que cursa com prejuízo da oxigenação do sangue, em virtude de alterações anatômicas no coração e/ou grandes vasos. Clinicamente pode apresentar-se com quatro alterações anatômicas importantes: estenose da artéria pulmonar, comunicação interventricular, dextroposição da aorta e hipertrofia do ventrículo direito. O Brasil acompanha a prevalência mundial de cardiopatias congênitas no período neonatal que gira em torno de $0,5 \%$ a $1 \%$, numa proporcionalidade oscilando de 8 a 10 para cada 1000 nascidos vivos, sem contar com os casos não diagnosticados por falta de triagem neonatal precoce. 0 tratamento da Tetralogia de Fallot é cirúrgico, para que haja 0 restabelecido da homeostasia anatomofisiológica do coração. Mas apesar da modernização das técnicas cirúrgicas atuais, as complicações pós-operatórias ainda apresentam morbidade elevada na população pediátrica e neonatal. As complicações, principalmente, pulmonares resultam em inúmeras intervenções fisioterapêuticas visando promover a higiene brônquica, maior sincronismo toracoabdominal, melhora da oxigenação e ventilação pulmonar, vias aéreas pérvias, melhora dos volumes e capacidades pulmonares. 0 objetivo do presente estudo é realizar uma revisão de literatura para fazer um levantamento das complicações mais frequentes e técnicas de assistência fisioterapêutica no pós-operatório de cirurgia cardíaca pediátrica de Tetra re Fallot. Para isso, foi realizada uma pesquisa be Tetralo (PEDro), e PubMed, utilizando as palavras-chave de acordo com a classificação dos Descritores em Ciências da Saúde (DCS): 'Fisioterapia'; 'Tetralogia de Fallot'; 'Cirurgia Cardíaca'; e 'Criança' As complicações pulmonares mais evidentes no pós-operatório da Tetralogia de Fallot foram pneumonia e atelectasia. Sendo a intervenção fisioterapêutica a melhor estratégia para otimiza a função pulmonar e minimizar estas complicações, através da assistência como suporte ventilatório invasivo protetor, desmame precoce, oxigenioterapia, mobilização precoce, posicionamento no leito, posição prona, AFE, hiperinsuflação manual, aspiração de vias aéreas, estímulo à tosse, ciclo ativo da respiração, técnica de expiração forçada, incentivadores inspiratórios, entre outras.

\section{Physiotherapy in the postoperative period of Tetralogy of Fallot}

Tetralogy of Fallot is a cyanotic congenital heart disease that causes damage to blood oxygenation due to anatomical changes in the heart and / or large vessels. Clinically it can present with four important anatomical changes: pulmonary artery stenosis, ventricular septal defect, aortic dextroposition and right ventricular hypertrophy. Brazil follows the worldwide prevalence of congenital heart disease in the neonatal period, which ranges from $0.5 \%$ to $1 \%$, with a proportionality ranging from 8 to 10 for every 1000 live births, not counting the cases not diagnosed for lack of neonatal screening precocious. The treatment of Tetralogy of Fallot is surgical, so that the anatomical and physiological homeostasis of the heart can be reestablished. But despite the modernization of current surgical techniques, postoperative complications still present high morbidity in the pediatric and neonatal population. Pulmonary complications result in numerous physiotherapeutic interventions aimed at promoting bronchial hygiene, increased thoracoabdominal synchronization, improved oxygenation and pulmonary ventilation, paten airways, improved pulmonary volumes and capacities. The objective of the present study is to perform a review of the literature to make a survey of the most frequent complications and techniques of physical therapy assistance in the postoperative period of pediatric cardiac surgery of Tetralogy of Fallot. For this, a bibliographic research was carried out in articles published in the last ten years in databases such as Scientific Electronic Library Online (SciELO), Latin American Literature in Health Science (LILACS), Google Academic, Coordination of Improvement of Higher Education Personnel (CAPES), Physiotherapy Evidence Database (PEDro), and PubMed, using the keywords according to the classification of Health Sciences Descriptors (DCS): 'Physiotherapy'; 'Tetralogy of Fallot'; 'Cardiac surgery'; and 'Child'. The most evident pulmonary complications in the postoperative period of Tetralogy of Fallot were pneumonia and atelectasis. As the physiotherapeutic intervention is the best strategy to optimize pulmonary function and minimize these complications, through assistance such as protective invasive ventilatory support, early weaning, oxygen therapy, early mobilization, bed positioning, prone position, AFE, manual hyperinflation, airway aspiration, cough stimulus, active cycle of breathing, forced expiration technique, inspiratory stimulators, among others.

Keywords: Physiotherapy; Tetralogy of Fallot; Cardiac surgery; Kid.

\section{Topic: Fisioterapia}

Reviewed anonymously in the process of blind peer.

Daniela Maristane Vieira Lopes Maciel (it)

Faculdade Guaraí, Brasil

http://lattes.cnpq.br/0591590391105455

http://orcid.org/0000-0001-5934-0219

daniela.marciel@iescfag.edu.br
Received: 14/08/2019

Approved: 22/08/2019
Referencing this:

MACIEL, D. M. V. L.. Fisioterapia no pós-operatório de Tetralogia de

Fallot. Scire Salutis, v.8, n.2, p.88-97, 2018. DOI: http://doi.org/10.6008/CBPC2236-9600.2018.002.0009

DOI: $10.6008 / C B P C 2236-9600.2018 .002 .0009$ 


\section{INTRODUÇÃO}

A Tetralogia de Fallot é uma cardiopatia congênita cianótica que se caracteriza por alterações anatômicas no coração e/ou grandes vasos (VASCONCELOS et al., 2014) com repercussões hemodinâmicas importantes ou potencialmente importantes (ROSA et al., 2013). Apresenta-se com quatro alterações anatômicas importantes: Estenose pulmonar: decorrente do fluxo sanguíneo diminuído pelo estreitamento da válvula pulmonar na saída do ventrículo direito.

Outras repercussões a serem destacadas são: Comunicação intraventricular (CIV): decorrente de defeito no fechamento do septo interventricular, produzindo mistura dos sangues arterial e venoso e queda das pressões internas; Dextroposição da aorta: decorrente da alteração do posicionamento da aorta, arranjando-se anatomicamente mais à direita; e Hipertrofia do ventrículo direito: decorrente do aumento da força de contração do ventrículo direito para bombear o sangue para artéria pulmonar mais estreita (O'BRIEN et al., 2014).

O Brasil acompanha a prevalência mundial de cardiopatias congênitas no período neonatal que gira em torno de $0,5 \%$ a $1 \%$ (CORREIA et al., 2015), numa proporcionalidade oscilando de 8 a 10 a cada 1000 nascidos vivos, sem contar com os casos não diagnosticados por falta de triagem neonatal precoce (ROSA et al., 2014; ALBUQUERQUE et al., 2015; MATHUR et al., 2018). Aproximadamente nascem no mundo por ano 1,35 milhão de crianças com cardiopatia congênita crítica, sendo de $6 \%$ a 10\% desse percentual associado a óbitos neonatais. Cerca de $25 \%$ das cardiopatias congênitas se manifestam nos primeiros meses após o nascimento com potencial risco de vida (MATHUR et al., 2017).

Dentre as malformações congênitas, as cardiopatias congênitas assumem a causa mais evidente de morte infantil, com prevalência de cerca de $40 \%$ no Brasil (JÚNIOR et al., 2015) e de $20 \%$ a $40 \%$ nos EUA (MATHUR et al., 2017), e as complicações decorrentes das cardiopatias como atrasos no desenvolvimento e déficits cognitivos englobam índices de $20 \%$ a 30\%. Estudos sobre a prevalência mundial estimam uma taxa de prevalência de 9,1 para cada 1000 nascimentos, após análise de 24.091 .867 nascimentos em 114 estudos de revisão e metanálise, números que se mostraram constantes nos últimos 15 anos (JÚNIOR et al., 2015).

A etiologia das cardiopatias congênitas incluindo a Tetralogia de Fallot são atribuídas a vários fatores, como aumento da idade materna, doenças durante a gestação, aumento da exposição a medicamentos como anticonvulsivantes, álcool e esteroides durante a gravidez e exposição ambiental a produtos químicos como solventes orgânicos, diclorodifeniltricloroetano (MATHUR et al., 2017). Os sinais clínicos evidentes na Tetralogia de Fallot manifestam-se precocemente nos primeiros meses de vida e apresentam-se com eventos de cianose, principalmente no período matutino ou desencadeados pela alimentação, desidratação, stress ou choro, acompanhada de alterações no padrão respiratório com taquipneia (O'BRIEN et al., 2014).

A abordagem de tratamento leva em consideração patologias associadas e intensidade das manifestações clínicas na criança que vão desde a ocorrência de cianose grave, dependente do canal arterial para sobreviver, até o assintomático. Existem duas possibilidades de intervenções cirúrgicas: uma paliativa e outra reparadora (CAMPOS et al., 2014). Mas apesar da modernização das técnicas cirúrgicas recentes, as 
complicações pós-operatórias ainda apresentam elevada morbidade nesta população neonatal e pediátrica (CATRÉ et al., 2013).

Os óbitos decorrentes de cirurgias cardíacas estão frequentemente associados às complicações pulmonares, cerca de $6 \%$ a $76 \%$, em função da própria particularidade do procedimento cirúrgico como anestesia geral, esternotomia, circulação extracorpórea (CEC), intensidade da manipulação cirúrgica e número de drenos, tempo de isquemia, deslocamento cefálico do diafragma causado pela anestesia, bloqueadores neuromusculares que levam ao colapso da função pulmonar, oxigenioterapia em excesso administrado durante a ventilação mecânica (BORGES et al., 2010; OLIVEIRA et al., 2013).

Por isso, a assistência fisioterapêutica é necessária para reverter ou prevenir as complicações, principalmente, pulmonares resultantes do procedimento cirúrgico, estando o lactente ou criança em suporte ventilatório invasivo ou respirando em ar ambiente, visando promover a higiene brônquica, manter as vias aéreas pérvias, melhorar da oxigenação e ventilação pulmonar, maior sincronismo toracoabdominal, otimizar volumes e capacidades pulmonares, aumentar o gradiente de pressão transpulmonar (SILVA et al., 2011).

Apesar de trabalhos que denotam os efeitos expressivos da fisioterapia no pós-operatório, são escassos estudos que agregam complicações e técnicas fisioterapêuticas no pós-operatório de cirurgia cardíaca pediátrica, sobretudo na Tetralogia de Fallot. O objetivo deste estudo é realizar uma revisão de literatura para levantar as complicações mais frequentes e técnicas de intervenção fisioterapêutica no pósoperatório de cirurgia cardíaca pediátrica de Tetralogia de Fallot.

\section{METODOLOGIA}

Trata-se de uma pesquisa bibliográfica em artigos publicados nos últimos dez anos, em bases de dados como Scientific Electronic Library Online (SciELO), Literatura Latina Americana em Ciência de Saúde (LILACS), Google Acadêmico, Coordenação de Aperfeiçoamento de Pessoal de Nível Superior (CAPES), Physiotherapy Evidence Database (PEDro), PubMed, entre os anos de 2008 e 2018. Utilizando as palavraschave de acordo com a classificação dos Descritores em Ciências da Saúde (DCS): 'Fisioterapia'; 'Tetralogia de Fallot'; 'Cirurgia Cardíaca'; e seus correlatos em inglês: 'Physiotherapy', 'Tetralogy of Fallot', 'Cardiac Surgery'.

\section{DISCUSSÃO TEÓRICA}

\section{Complicações Pulmonares}

Alguns eventos contribuem para a deterioração da função pulmonar e o desencadeamento de cascata inflamatória tais quais: esternotomia, anestesia geral, circulação extracorpórea (CEC), suporte ventilatório invasivo, intensidade da manipulação cirúrgica, número de drenos, tempo de isquemia (BORGES et al., 2010), oxigenioterapia em excesso durante a ventilação mecânica, bloqueadores neuromusculares, deslocamento cefálico do diafragma causado pela anestesia (OLIVEIRA et al., 2013). 
A utilização de anestesia é um dos fatores que acarretam os distúrbios de ventilação-perfusão, reduzindo a CRF em torno de $20 \%$, ligando tal fato à atelectasia secundária e ao fechamento das vias aéreas distais. Ainda, a dor no pós-operatório e a presença dos drenos resultam em baixos volumes pulmonares (SOARES et al., 2017).

A CEC é uma modo de circulação artificial, utilizada na maioria das cirurgias cardíacas corretivas, e cursa com lesão pulmonar, deflagrando uma cascata de ativação inflamatória por meio de ativação celular e liberação de mediadores inflamatórios (SOARES et al, 2010). As cirurgias cardíacas associadas à CEC geram um acúmulo de líquidos intrapulmonar, congestão capilar, colapso alveolar, processo inflamatório intenso, prejuízo das trocas gasosas, redução da complacência pulmonar, redução dos volumes e capacidades pulmonares, atelectasias e hipoxemia (RODRIGUES et al., 2010; PADOVANI et al., 2011).

Algumas complicações pulmonares como atelectasia, derrame pleural, hipoxemia, pneumomediastino, lesão pulmonar e ou laringite foram identificados no estudo realizado por Oliveira et al. (2012) em um hospital universitário brasileiro, com revisão de 55 prontuários retrospectivamente, de crianças que realizaram correção cardíaca cirúrgica, em torno de 16,5\% com diagnóstico de Tetralogia de Fallot. Com relação ao procedimento cirúrgico 35 crianças precisaram de CEC, tempo médio de 34 minutos (mínimo de 20 e máximo de 165 minutos). No pós-operatório, os pacientes precisaram de suporte ventilatório invasivo por um tempo médio de 10 horas, na modalidade ventilação mandatória intermitente sincronizada (SIMV) e 53\% desenvolveram complicações pulmonares.

As complicações pulmonares podem causar também desvio para o metabolismo anaeróbico com evidencia de acidose metabólica, aumento no nível de lactato e circulação microvascular prejudicada, que podem ser solucionados ou prevenidos por meio da assistência fisioterapêutica (OLIVEIRA et al., 2018). Um estudo de corte retrospectivo realizado por BORGES e colaboradores, em 2010, numa população amostral de 37 crianças com idade inferior a 12 anos, observou que 70,3\% dos que necessitaram de CEC durante procedimento cirúrgico, por um período oscilando entre 25 e 180 minutos, apresentaram complicações pulmonares num percentual de 7,6\%. Necessitaram de ventilação mecânica no pós-operatório 64,9\% dos pacientes, com tempo oscilando entre 1 e 362 horas, média de 6,6 horas, e desenvolveram complicações pulmonares cerca de $8,3 \%$.

O ensaio clínico aleatório realizado por FELCAR e colaboradores, em 2008, no Hospital Infantil Sagrada Família, no Paraná, com 135 pacientes de 0 a 6 anos no pós-operatório de cirurgia cardíaca identificou a presença de pneumonia e atelectasia como complicações pulmonares mais evidentes. $\mathrm{O}$ grupo controle que realizou fisioterapia apenas nos pós-operatório desenvolveu pneumonia na proporção de 19,4\%, atelectasia $11,9 \%$ e pneumonia e atelectasia $11,9 \%$. Outras complicações também foram evidenciadas em menor proporção como derrame pleural, hemorragia pulmonar, hipertensão pulmonar, quilotórax, pneumotórax e paralisia diafragmática.

Os critérios para identificação dessas complicações respiratórias no estudo de Oliveira et al. (2012), foram: derrame pleural, atelectasia e pneumomediastino, imagem radiológica anormal acompanhada de sintomas respiratórios agudos; pneumonia, presença de imagem radiológica com infiltrado pulmonar 
recente associado à secreção traqueobrônquica purulenta com leucocitose e febre; e laringite, presença de estridor inspiratório associado ao desconforto respiratório agudo. Os percentuais obtidos foram 31\% dos pacientes evoluíram com derrame pleural, atelectasia e 5,5\% laringite, pneumomediastino, lesão pulmonar, nenhum paciente desenvolveu pneumonia e $24 \%$ das crianças apresentaram alterações cardíacas e renais.

Campos et al. (2014), realizou um estudo de corte retrospectivo com 13 crianças, com diagnóstico confirmado de Tetralogia de Fallot, que no pós-operatório apresentaram complicações de pneumonia, hemotórax, taquicardias juncionais ectópicas e convulsões. As sequelas desenvolvidas tardiamente foram dilatação ventricular direita e insuficiência pulmonar moderada a severa com $77,8 \%$ e insuficiência tricúspide em $66,7 \%$. As alterações nas áreas motoras e da linguagem também foram identificadas mais tardiamente.

A atelectasia é o colapso de áreas do parênquima pulmonar, gerando o colabamento das vias aéreas, que evolui com deterioração da oxigenação, prejuízo da mecânica respiratória, redução da capacidade residual funcional, diminuição da complacência pulmonar, inibição do reflexo de tosse, redução da higiene brônquica, levando à insuficiência respiratória e aumento da resistência vascular pulmonar (CAVENAGHI, et. al., 2009) e queda na produção do surfactante. As crianças estão no grupo de risco, pois apresentam atelectasia de forma mais frequente, devido à reduzida CRF e complacência da caixa torácica elevada (PINTO et al., 2015).

A pneumonia é uma infecção do trato respiratório inferior, que compromete as vias aéreas e o parênquima, com ou sem consolidação dos espaços alveolares (MACHADO et al., 2010). A pneumonia associada ao suporte ventilatório invasivo é muito comum na UTI, aumenta a incidência com o tempo duração da ventilação artificial, cerca de 3\% por dia, porém é um evento prevenível (DALMORA et al., 2013).

\section{Intervenção Fisioterapêutica no Pós-Operatório}

Pelo elevado risco de complicações e todo processo advindo da cirurgia cardíaca a intervenção fisioterapêutica no pré e pós-operatório das cirurgias cardíacas em crianças ajudam a manter as vias aéreas pérvias, melhoram a ventilação pulmonar, facilitam as trocas gasosas, promovem a remoção de secreções pulmonares, assim como o seu acúmulo, auxiliam na distribuição do fluxo aéreo de forma adequada, melhoram e mantem os volumes e capacidades pulmonares dentro da normalidade, melhoram a complacência e resistência pulmonar, estimulam a deambulação precoce do paciente. Além de contribuírem para a diminuição do tempo de permanência na UTI, o tempo de internação hospitalar e consequentemente, diminuir as complicações pulmonares (SILVA et al., 2011), e facilitar a extubação precoce (RIBEIRO et al., 2008; CAVENAGHI et al., 2009).

As cirurgias cardíacas pediátricas possuem peculiaridades próprias e representam a associação entre os achados clínicos, novas técnicas cirúrgicas e avanços tecnológico, com o objetivo de minimizar as complicações em crianças. A integração do fisioterapeuta na equipe multidisciplinar no pré, peri e pósoperatório contribuem para minimizar, prevenir e tratar as complicações pulmonares decorrentes do procedimento cirúrgico e gravidade da patologia. Selecionar a estratégia de intervenção fisioterapêutica adequada em cada momento vai emanar a integração de conhecimento de fisiopatologia da cardiopatia, tipo 
de cirurgia, reparadora ou paliativa, gravidade da doença e a própria condição clínica da criança (SILVA et al., 2011).

As crianças, sobretudo até os 3 anos de idade, apresentam a parede torácica muito complacente, contração do diafragma pouco efetiva predispondo a distorções torácicas e ocorrência de assincronismo. Em contrapartida a complacência pulmonar é menor, levando a redução da capacidade residual funcional (CRF) em relação aos adultos. Com isso são mais vulneráveis à fadiga muscular, obstrução das vias aéreas inferiores e atelectasias, principalmente quando eles são submetidos a efeito anestésico (PINTO et al., 2015).

A fisioterapia no pré-operatório é um fator importante e serve como uma estratégia preventiva de orientação aos pais das crianças sobre os benefícios da fisioterapia, procedimentos e técnicas utilizadas para a recuperação da função pulmonar e precocidade da alta hospitalar (FELCAR et al., 2008). Além de realização de manobras desobstrutivas, reexpansivas e sincronismo toracoabdominal (CAVENAGHI et al., 2009).

No pós-operatório as técnicas fisioterapêuticas utilizadas são: ciclo ativo da respiração, aceleração do fluxo expiratório, expiração forçada, incentivadores inspiratórios, exercícios respiratórios, ventilação mecânica não invasiva (VNI), tosse, drenagem postural (SILVA et al., 2011), vibrocompressão manual, aspiração de vias aéreas (CAVENAGHI et al., 2009; SILVA et al., 2011; ASSUPÇÃO et al., 2013), RPPI (pressão positiva intermitente nas vias aéreas ) (COSTA, 2013), mobilização no leito precocemente, posicionamento correto no leito (RODRIGUES et al., 2010), reequilíbrio toracoabdominal, bag squeezing (RODRIGUES et. al., 2010), manobras de reexpansão pulmonar (PADOVANI et al., 2011), posição prona (RIBEIRO et al., 2008), fortalecimento da musculatura respiratória (LACERDA et al., 2013).

A aplicação das técnicas fisioterapêuticas no pós-operatório de cirurgias cardíacas apresentam algumas particularidades, pois, manobras bruscas são restritas para os casos que evoluem com hipertensão pulmonar, em função da adaptação resistência vascular pulmonar nesse período (SILVA et al., 2011). A assistência fisioterapêutica tem início quando o lactente ou criança da entrada na UTI, o profissional deve garantir um bom posicionamento no leito, ajustar parâmetros do suporte ventilatório invasivo, avaliar prontuário com informações do período intraoperatório.

Além disso, ajudar a equipe de enfermagem na acomodação da cânula traqueal para evitar risco de extubação acidental, acessos vasculares e drenos, avaliar gasometria arterial e imagens radiológicas no pósoperatório imediato e iniciar a fisioterapia respiratória com manobras de higiene brônquica e reexpansão pulmonar (SILVA et al., 2011), além de ajustar frações de oxigênio menor possível, pois altas concentrações de oxigênio também induzem a atelectasia e a ocorrência de shunt pulmonar (PINTO et al., 2015).

Um estudo realizado por Ultra et al. (2010) analisou a intervenção fisioterapêutica em uma UTI no Rio de Janeiro, em lactentes de 0 a 2 anos, no pós-operatório imediato de cirurgia de Tetralogia de Fallot, para correção de dextroposição da aorta, os resultados mostraram-se positivos na prevenção de complicações como atelectasias e derrame pleural, garantindo melhora da função pulmonar através da higiene brônquica, reexpansão pulmonar, estabilização hemodinâmica dos sinais vitais, melhora da saturação de oxigênio, redução do esforço respiratório e tempo de permanência na UTI. A adequação dos 
parâmetros ventilatórios devem ser de acordo idade da criança, peso corporal e condição clínica, após avaliação prévia de gasometria arterial, imagem radiológica e parâmetros hemodinâmicos.

Deve-se preconizar parâmetros que garantam uma ventilação protetora e previnam o colapso alveolar ajustando uma menor fração inspirada de Oxigênio $\left(\mathrm{FiO}_{2}\right)$ apenas para garantir saturação arterial de oxigênio adequada, volume corrente entre 6 e $8 \mathrm{ml} / \mathrm{kg}$, pressão parcial de oxigênio $\left(\mathrm{PaO}_{2}\right)$ entre $80-90 \mathrm{mmHg}$, pressão positiva expiratória final (PEEP) entre $5-6 \mathrm{mmHg}$, pressão inspiratória necessária para manter valores de pressão parcial de gás carbônico $\left(\mathrm{PaCO}_{2}\right)$ entre $35-45 \mathrm{mmHg}$ e tempo inspiratório adequado. Deve-se ajustar os parâmetros em consonância com variações hemodinâmicas que aconteceram em função da correção cirúrgica para manter um retorno venoso adequado e evitar hipo ou hiperfluxo pulmonar (COSTA, 2013).

A maioria das cardiopatias congênitas cursam com falência do ventrículo direito, que podem piora com o aumento de pressões transtorácicas, evitando diminuição acentuada do retorno venoso e deterioração do débito cardíaco, sendo assim o profissional deve ter cautela e ajustar a PEEP e Pressão inspiratória observando a monitorização hemodinâmica e condição clínica da criança (ABUD, 2015).

Outro ponto importante a considerar é manter o suporte ventilatório invasivo pelo menor tempo possível e cessá-lo tão logo diminua o efeito da anestesia, procedendo com o desmame evitando assim pneumonias, hipertrofia do diafragma e aumento da morbimortalidade com o uso prolongado da ventilação artificial (SILVA et al., 2008). A decisão de iniciar o desmame ventilatório deve ser pautada na estabilidade clínica e hemodinâmica, gasometria arterial, nível de consciência (JOHNSTON et al., 2008), ausência de sinais de desconforto respiratório como batimentos de asa de nariz, tiragens e taquipneia (SILVA et al., 2008). Mesmo não havendo preditores que garantam o sucesso absoluto da extubação, esta deve ser iniciada precocemente. Atualmente, em algumas UTIs, tem sido preconizada na admissão do lactente ou criança na unidade (JOHNSTON et al., 2008).

O estudo controlado e randomizado realizado por Assumpção et al. (2013), na UTI cardiológica do Hospital Pequeno Príncipe, Curitiba (PR), analisou o efeito de manobras de higiene brônquica em 20 lactentes extubados no pós-operatório de cardiopatia congênita, entre 0 e 12 meses, em 2 grupos, apresentando estabilidade hemodinâmica, sem sedação ou efeito analgésico. Evidenciando que a utilização de técnicas fisioterapêuticas como a vibrocompressão manual e a aspiração tiveram efeito positivo em lactentes sem causar alterações da saturação de oxigênio e frequência respiratória, nem desencadear dor e desconforto respiratório. As manobras de higiene brônquica como hiperinsuflação manual, associada ou não a vibrocompressão, AFE lento e rápido são recomendadas para descolar e mobilização da secreção das vias aéreas em pediatria e neonatologia (JOHNSTON et al. 2012).

A percussão torácica não é recomendada durante ventilação artificial ou pós-extubação em neonatos, pois, podem resultar no aumento da pressão intratorácica e causar hipoxemia. Quando realizada de forma rotineira com tempo duração de 1 a 2 minutos em recém-nascidos com idade gestacional entre 28 a 37 semanas, imediatamente após a extubação, pode ocasionar o colabamento alveolar (JOHNSTON et al., 2012). 
Técnicas de reexpansão pulmonar para reverter ou prevenir complicações como atelectasia e redução da CRF, incluem o aumento da pressão sustentada por múltiplos ou um único período de tempo; o aumento gradual ou progressivo da PEEP, este podendo alterar ou não o volume corrente; aumento da PEEP com alteração ou manutenção da pressão controlada; suspiros intermitentes; posição prona no leito; ventilação de alta-frequência (VAFO) muito utilizada em neonatologia; aumento do tempo inspiratório e inversão da relação inspiração-expiração. Os métodos mais utilizados na população pediátrica são a insuflação sustentada, insuflação gradual, Posição prona e suspiros intermitentes (CROSARA et al., 2013).

A posição prona vem ganhando atenção de pesquisadores e sendo indicada também no pósoperatório de CC para proporcionar conforto respiratório na ocorrência de SARA (Síndrome do Desconforto Respiratório Agudo), melhorar da perfusão sanguínea, redistribuição da ventilação alveolar, melhora da relação ventilação/perfusão (V/Q), melhora dos volumes e capacidades pulmonares, equilíbrio e sincronismo toracoabdominal (RIBEIRO et al., 2008), eficácia da relação $\mathrm{PaO}_{2} / \mathrm{FiO}_{2}$ (RODRIGUES et al., 2010), reexpandi áreas mais dorsais do pulmão onde o colapso alveolar é mais predominante (CROSARA et al., 2013).

O posicionamento em decúbito lateral e sua alternância viabiliza a redistribuição ventilatória canalizando o fluxo aéreo para o pulmão não-dependente, contribuindo para a reexpansão de áreas atelectasiadas (RIBEIRO et al., 2008). Além de beneficiar as estruturas musculoesqueléticas prevenindo úlceras de pressão, encurtamentos, neuropatias de compressão e fenômenos tromboembólicos (VECCHIO et al., 2015), estimulação a micro e macrocirculação (SOARES et al., 2017).

A reexpansão pulmonar é imprecisndível no pós-operatório de cirurgia cardíaca em crianças com suporte ventilatório invasivo com o objetivo de promover um aumento da pressão transpulmonar, garantindo a abertura dos alvéolos e aumento das áreas de trocas gasosas, reduzindo a hipoxemia. Sendo a técnica mais indicada a insuflação sustentada, que consiste na utilização do CPAP com pressões oscilando entre 30 a $40 \mathrm{cmH}_{2} \mathrm{O}$, por 30-40 segundos (NEVES et al., 2009; SANTOS et al., 2011; PINTO et al., 2015). A contraindicação para as manobras de reexpansão alveolar aplica-se a neonatos e crianças com instabilidade hemodinâmica, com presença de hipertensão intracraniana e pneumotórax não drenado (NEVES et al., 2009).

Os exercícios respiratórios devem ser realizados a depender do nível de consciência e colaboração da criança, pois melhoram a função pulmonar, aumentam o calibre das vias aéreas, facilitam a remoção de secreções das vias áreas periféricas e facilitam a expansão pulmonar (SILVA et al., 2011). A mobilização precoce deve ser preconizada desde o período de sedação através da mobilização passiva, a fim de garantir a integridade das articulações, estimular a ativação cortical e otimizar a recuperação funcional. Na sequência serão introduzidos exercícios ativo assistido, ativo livre, sedestação, ortostatismo, exercícios transferência e deambulação precoce assistida e independente para as crianças que já apresentam marcha independente (VECCHIO et al., 2015).

A mobilização precoce deve estimulada com cautela, uma vez que existe uma baixa tolerância ao exercício em crianças com Tetralogia de Fallot, estenose aórtica grave, coarctação da aorta com hipertensão arterial e no ventrículo único após cirurgia de Fontan. Em geral, aquelas que apresentam cardiopatias mais 
complexas apresentando incompetência cronotrópica, baixo duplo produto de pico, baixo consumo máximo de oxigênio e arritmias ventriculares durante a avaliação funcional ao exercício (PFEIFFER, 2009).

Deve, o fisioterapeuta, estar atento a sinais da síndrome de baixo débito cardíaco como sudorese, sinais de agitação psicomotora, extremidades frias, lábios pálidos, pulsos periféricos ausentes ou filiformes, hipotensão e oligúria (SILVA et al., 2011). Após estabilidade respiratória e hemodinâmica do neonato ou criança ele receberá alta da UTIN e será encaminhado para enfermaria e continuará ser acompanhado pelo fisioterapeuta no pós-operatório tardio (ABUD, 2011).

\section{CONSIDERAÇÕES FINAIS}

As complicações pulmonares mais evidentes no pós-operatório da Tetralogia de Fallot foram pneumonia e atelectasia. Sendo a intervenção fisioterapêutica a melhor estratégia para otimizar a função pulmonar e minimizar estas complicações, através da assistência como suporte ventilatório invasivo protetor, desmame precoce, ajustes de parâmetros ventilatórios invasivos e não invasivos, oxigenioterapia, mobilização precoce, posicionamento no leito, posição prona, AFE, hiperinsuflação manual, aspiração de vias aéreas, estímulo a tosse, ciclo ativo da respiração, técnica de expiração forçada, incentivadores inspiratórios e exercícios respiratórios. Ainda existe uma lacuna quanto as evidências científicas para técnicas fisioterapêuticas no pós-operatório de cirurgia cardíaca na Tetralogia de Fallot em neonatologia e pediatria e mais ensaios clínicos devem ser realizados.

\section{REFERÊNCIAS}

ABUD, K. C. O.. Cardiopatias congênitas. In: SARMENTO, G. J. V.. Fisioterapia respiratória em pediatria e neonatologia. 2 ed. Barueri: Manole, 2011. p.166-179.

ALBUQUERQUE, F. C. L.; MAIA, E. T.; FIGUEIREDO, V. L.; MOURATO, F. A.; MATTO, S. S.. Exame Físico e Oximetria de Pulso para Detectar Cardiopatias Congênitas. Internacional Journal of Cardiovascular Sciences, Rio de Janeiro, v.28, n.2, p.148-151, 2015. DOI: http://doi.org/10.5935/2359$\underline{4802.20150023}$

ASSUMPÇÃO, M. S.; GONÇALVES, R. M.; KRYGIEROWICZ, L. C.; ORLANDO, A. C. T.; SCHIVINSKI, C. I. S.. Vibrocompressão manual e aspiração nasotraqueal no pós-operatório de lactentes cardiopatas. Revista Paulista de Pediatria, São Paulo, v.31, n.4, p.507-515, 2013.

BORGES, D. L.; SOUSA, L. R. T.; SILVA, R. T.; GOMES, H. C. R.; FERREIRA, F. M. M.; LIMA, W. L.; BORGES, L. C. P. L.. Complicações pulmonares em crianças submetidas à cirurgia cardíaca em um hospital universitário. Revista Brasileira de Cirurgia Cardiovascular, São Paulo, v.25, n.2, p.234-237, 2010.

CAMPOS, M.; RODRIGUES, M.; MOURA, C.; GUIMARÃES, H.. Tetralogia de Fallot: uma cardiopatia com fisiopatologia e evolução variáveis. Dissertação (Mestrado Integrado em Medicina) - Universidade do Porto, Porto, 2014.

CATRÉ, D.; LOPE, M. F.; MADRIGAL, A.; OLIVEIROS, B.; CABRITA A. S.; VIANA, J. S.; NEVES, J. F.. Fatores preditivos de complicações graves em cirurgia neonatal. Revista do Colégio Brasileiro de Cirurgiões, Rio de Janeiro, v.40, n.5, p.363-369, 2013.

CAVENAGHI, S.; MOURA, S. C. G.; SILVA, T. H.; VENTURINELLI, T. D.; MARINO, L. H. C.; LAMARI, N. M. Importância da fisioterapia no pré e pós-operatório de cirurgia cardíaca pediátrica. Revista Brasileira de Cirurgia Cardiovascaular, São Paulo, v.24, n.3, p.397-400, 2009.

CORREIA, M.; FORTUNATO, F.; MARTINS, D.; TEIXEIRA, A.; NOGUEIRA, G.; MENEZES, I.; ANJOS, R.. Cardiopatias Congénitas Complexas: Influência do Diagnóstico PréNatal. Acta Medica Portuguesa, Lisboa, v.28, n.2, p.158163, 2015.

DALMORA, C. H.; DEUTSCHENDORF, C.; NAGEL, F.; SANTOS, R. P.; LISBOA, T.. Definindo pneumonia associada à ventilação mecânica: um conceito em (des)construção. Revista Brasileira de Terapia Intensiva, São Paulo, v.25, n.2, p.81-86, 2013

FELCAR, J. M.; GUITTI, J. C. S.; MARSON, A. C.; CARDOSO, J. R.. Fisioterapia pré-operatória na prevenção das complicações pulmonares em cirurgia cardíaca pediátrica. Revista Brasileira de Cirurgia Cardiovascular, São Paulo, v.23, n.3, p.383-388, 2008.

JOHNSTON, C.; PIVA, J. P.; CARVALHO, W. B.; GARCIA, P. C.; FONSECA, M. C.; HOMMERDING, P. X.. Preditores de Falha da Extubação em Crianças no Pós-Operatório de Cirurgia 
Cardíaca Submetidas à Ventilação Pulmonar Mecânica. Revista Brasileira de Terapia Intensiva, São Paulo, v.20, n.1, p.57-62, 2008.

JOHNSTON, C.; ZANETTI, N. M.; COMARU, T.; RIBEIRO, S. N. S.; ANDRADE, L.B.; SANTOS, S. L. L.. Recomendação brasileira de fisioterapia respiratória em unidade de terapia intensiva pediátrica e neonatal. Revista Brasileira de Terapia Intensiva, São Paulo, v.24, n.2, p.119-129, 2012.

JÚNIOR, V. C. P.; BRANCO, K. M. P. C.; CAVALCANTE, R. C.; JUNIOR, W. C.; LIMA, J. R. C.; FREITAS, S.. M.; FRAGA, M. N. O.; SOUZA, N. M. G.. Epidemiologia da cardiopatia congênita no Brasil. Brazilian Journal of Cardiovascular Surgery, São Paulo, v.30, n.2, p.219-224, 2015.

LACERDA, A. A.; SILVA, B. R. B; FILHO, A. A. S.; SILVA, E. F. R.. Tetralogia de Fallot: Aspectos clínicos, diagnósticos e terapêuticos. Revista Multiprofissional em Saúde do Hospital São Marcos, Teresina, v.1, n.1, p.50-57, 2013.

MACHADO, D.; MORAES, L. T. A. R.; ESCARELI, M. O.; VIANNA, I. A.. Pneumonia: Tratamento e Evolução. Cadernos UnifOA, Volta Redonda, v.5, n.14, p.65-69, 2010.

NEVES, V; KOLISLI, A; GIRALDI, J.. A manobra de recrutamento alveolar em crianças submetidas à ventilação mecânica em unidade de terapia intensiva pediátrica. Revista Brasileira de Terapia Intensiva, São Paulo, v.21, n.4, p.453-460, 2009.

O'BRIEN, P.; MARSHALL, A. C.. Tetralogy of Fallot. Cardiology Patente Page, v.130, p.26-29, 2014. DOI: http://doi.org/10.1161/circulationaha.113.005547

OLIVEIRA, P. M. N.; HELD, P. A.; GRANDE, R. A. A.; RIBEIRO, M. A. O.; BOBBIO, T. G.; SCHIVINSKI, C. I. S.. Perfil das crianças submetidas à correção de cardiopatia congênita e análise das complicações respiratórias. Revista Paulista de Pediatria, São Paulo, v.30, n.1, p.116-121, 2012.

OLIVEIRA, S. S.; MANSUETO NETO, A. R. A. S.; JUNIOR R.. Terapia de Expansão Pulmonar na Oxigenação Arterial e Nível Sérico de Lactato no Pós-Operatório de Cirurgia Cardíaca. International Journal of Cardiovascular Science, Rio de Janeiro, v.31, n.1, p.63-70, 2018. DOI: http://doi.org/10.5935/2359-4802.20170092

PADOVANI, C.; CAVENAGHI, O. M.. Recrutamento alveolar em pacientes no pós-operatório imediato de cirurgia cardíaca. Revista Brasileira de Cirurgia Cardiovascular, São Paulo, v.26, n.1, p.116-121, 2011.

PFEIFFER, M. E. T.. Reabilitação cardíaca pediátrica. Revista do DERC, n.47, p.18-20, 2009.

PINTO, A. S.; REIS, M.; TEIXEIRA C. L. C.; JÚNIOR, R. C.; LINO, A. P. B.. Recrutamento alveolar: em quem? Como? Quando? Revista de Medicina de Minas Gerais, Belo Horizonte, v.25, n.4, p.48-55, 2015.
RIBEIRO, I. F.; MELO, A. P. L.; DAVIDSON, J.. Fisioterapia em recém-nascidos com persistência do canal arterial e complicações pulmonares. Revista Paulista de Pediatra, São Paulo, v.26, n.1, p.77-83, 2008.

RODRIGUES, C. D. A.; OLIVEIRA, R. A. R. A.; SOARES, S. M. T. P.; FIGUEIREDO, S. A.; DRAGOSAVAC, D.. Lesão pulmonar e ventilação mecânica em cirurgia cardíaca: revisão. Revista Brasileira de Terapia Intensiva, São Paulo, v.22, n.4, p.375$383,2010$.

ROSA, M. P.; COSTA, P. O.; VASCONCELLOS, N. M.; DA SILVA, J. F.; BOFF, A.; DOS SANTOS, L. J.. Reabilitação cardiorrespiratória em crianças: uma revisão da literatura. ASSOBRAFIR Ciência, São Paulo, v.5, n.2, p.47-57, 2014.

ROSA, R. C. M.; ROSA, R. F. M.; ZEN, P. R.; PASKULIN, G. A.. Cardiopatias congênitas e malformações extracardícas. Revista Paulista de Pediatria, São Paulo, v.31, n.2, p.234251, 2013.

SANTOS, R. S.; SILVA, P. L.. Manobras de recrutamento: prós e contras. Pulmão RJ, Rio de Janeiro, v.20, n.3, p.7-12, 2011.

SILVA, M. E. M.; FEUSER, M. R.; SILVA, M. P.; UHLIG, S.; PARAZZI, P. L. F.; ROSA, G. J.; SCHIVINSKI, C. I. S.. Cirurgia cardíaca pediátrica: o que esperar da intervenção fisioterapêutica?. Revista Brasileira de Cirurgia Cardiovascular, São Paulo, v.26, n.2, p.264-272, 2011.

SILVA, Z. M.; PEREZ, A.; PINZONI, A. D.; RICACHINEWSKY, C. R.; RECH, D. R.; LUKRAFKA, J. L.. Fatores associados ao insucesso no desmame ventilatório de crianças submetidas a cirurgia cardíaca pediátrica. Revista Brasileira de Cirurgia Cardiovascular, São Paulo, v.23, n.4, p.501-506, 2008.

SOARES J. L.; SOUZA, A. M. B.; MEDEIROS, J. S.. Tratamento Fisioterapêutico no pré e pós-operatório de cirurgia cardíaca: revisão integrativa. Revista Ciência e Saberes, Caxias, v.3, n.3, p.624-629, 2017.

SOARES, L. C. C.; RIBAS, D.; SPRING, R.; SILVA, J. M. F.; MIYAGUE, N. I.. Perfil Clínico da Resposta Inflamatória Sistêmica após Cirurgia Cardíaca Pediátrica com Circulação Extracorpórea. Arquivos Brasileiros de Cardiologia, São Paulo, v.94, n.1, p.127-133, 2010.

ULTRA, R. B.; PERRUSO, C. A.; FERNANDES, E. T.. A atuação da Fisioterapia intensiva no pós-operatório imediato de correção da cardiopatia congênita tipo Tetralogia de Fallot. Revista Brasileira de Cardiologia, São Paulo, n.23, p.82-87, 2010.

VASCONCELOS, A. K.; ROCHA, F.. Atuação da fisioterapia em crianças submetidas à correção cirúrgica de Tetralogia de Fallot: uma revisão integrativa. EFDeportes.com, Buenos Aires, v.19, n.195, 2014

VECCHIO, D. V.; NOZAWA, E.. Mobilização do paciente crítico. In: FELTRIM, M. I. Z.; NOZAWA, E.; SILVA, A. M. P. R.. Fisioterapia Cardiorrespiratória na UTI Cardiológica. São Paulo: Blucher, 2015. p.238-251.

A CBPC - Companhia Brasileira de Produção Científica (CNPJ: 11.221.422/0001-03) detém os direitos materiais desta publicação. Os direitos referem-se à publicação do trabalho em qualquer parte do mundo, incluindo os direitos às renovações, expansões e disseminações da contribuição, bem como outros direitos subsidiários. Todos os trabalhos publicados eletronicamente poderão posteriormente ser publicados em coletâneas impressas sob coordenação da Sustenere Publishing, da Companhia Brasileira de Produção Científica e seus parceiros autorizados. Os (as) autores (as) posteriormente ser publicados em coletâneas impressas sob coordenação da Sustenere Publishing, da Companhia Brasileira de Produção Cientifica e seus parce
preservam os direitos autorais, mas não têm permissão para a publicação da contribuição em outro meio, impresso ou digital, em português ou em tradução. 